\title{
Primary Tuberculous Cerebellar Abscess in an Immunucompetent Adult
}

\author{
Okacha Naama ${ }^{1}$, Omar Boulahroud ${ }^{1}$, Mostapha Elouennass ${ }^{2}$, Ali Akhaddar ${ }^{1}$, \\ Miloudi Gazzaz ${ }^{1}$, Brahim Elmoustarchid ${ }^{1}$ and Mohamed Boucetta ${ }^{1}$
}

Key words: cerebellar abscess, tuberculous abscess, immunocompetent adult

(Inter Med 49: 875-876, 2010)

(DOI: 10.2169/internalmedicine.49.3397)


Picture 1. Enhanced CT-scan on axial view (Picture A) and sagittal MR T1-weighted image (Picture B) showing ring-enhancing mass with surrounding edema in the left cerebellar hemisphere.

A 48-year-old immunocompetent woman, with no past medical history of tuberculosis was admitted at the service with a one-month history of occipital headache, vomiting, and ataxic gait. Neurological evaluation showed signs of cerebellar dysfunction, including ataxia, wide-based gait, and left dysmetria. Routine full blood count with differential, liver function and renal function tests were all normal. Quantitative C-reactive protein was $7 \mathrm{mg} / \mathrm{L}$ (0.0-5.0 mg/L). HIV serology was negative. A tuberculin skin test was 25 $\mathrm{mm}$ at 48 hours and the chest X-ray was normal. CT-scan and brain MR-imaging revealed a ring-enhancing mass with surrounding edema in the left cerebellar hemisphere (Picture 1). The patient underwent a left suboccipital craniec- tomy and resection of a lobulated abscess, draining $5 \mathrm{~mL}$ of pus. Ziel-Neelsen staining of the abscess material revealed that acid-fast bacilli (AFB) were present (Picture 2). Histopathology showed an extensive inflammatory infiltrate, without granulomatous reaction, caseous necrosis and langhans cells. Polymerase chain reaction analysis revealed the presence of Mycobacterium tuberculosis. Antituberculous treatment including rifampicin, isoniazid, streptomycin and pyrazinamide was given. The patient made an uneventful recovery and is being continued on antituberculous chemotherapy.

Tuberculous brain abscess (TBA) is one of the rare forms of central nervous system tuberculosis. Cerebellar abscess

${ }^{1}$ Department of Neurosurgery, Mohammed V Military Teaching Hospital, Mohammed V-Souissi University, Rabat, Morocco and ${ }^{2}$ Department of Bacteriology, Mohammed V Military Teaching Hospital, Mohammed V-Souissi University, Rabat, Morocco

Received for publication January 17, 2010; Accepted for publication January 26, 2010

Correspondence to Dr. Okacha Naama, okacha_naama@hotmail.com 




Picture 2. Ziehl-Neelson staining of the abscess material revealed acid fast bacilli (stained red).

due Mycobacterium tuberculosis is extremely rare even in a country where tuberculosis is an alarming public health problem $(1,2)$. It is usually associated with foci of infection either in the lung or with an immunocompromised state (3). An isolate or primary TBA with no evidence of tuberculosis elsewhere is even rarer. The criteria for diagnosis include pus within the brain, the presence of AFB in the pus and the absence of caseation and granuloma formation (4). All of these criteria were met during diagnosis of cerebellar abscess in the present case. In the series reported by Chakraborti et al (5), of 21 cases of TBA, only three cases satisfied these criteria. The prevalence of TBA is increased in the third and fourth decades of life. There may be supratentorial abscesses, but rarely in cerebellum as observed in the present case $(3,5)$. Surgery combined with specific antituberculousis treatment seems to determine a good outcome (2).

\section{References}

1. Ramesh VG, Sundar KS. Concomitant tuberculous and pyogenic cerebellar abscess in a patient with pulmonary tuberculosis. Neurol India 56: 91-92, 2008.

2. Gazzaz M, Bouyaakoub FA, Elkhamlichi A. Tuberculous cerebellar abscess. Acta Neurol Belg 100: 146-147, 2000.

3. Wanjari K, Baradkar VP, Nataraj G, Kumar S. A rare case of tubercular cerebellar abscess. Indian J Med Microb 27: 363-365,
2009.

4. Ersoy Y, Ates O, Onal C, et al. Cerebellar abscess and syringomyelia due to isoniazid-resistant Mycobacterium tuberculosis. J Clin Neurosci 14: 86-89, 2007.

5. Chakraborti S, Mahadevan A, Govinda A, et al. Clinicopathological study of tuberculous brain abscess. Pathol Res Pract 205: 815$822,2009$.

(C) 2010 The Japanese Society of Internal Medicine http://www.naika.or.jp/imindex.html 